\title{
Instantaneous micropatterning of a double-network hydrogel surface triggered by mechanical force
}

\section{Qifeng Mu}

Hokkaido University https://orcid.org/0000-0003-3509-429X

Kunpeng Cui

Hokkaido University

Zhi jian Wang

https://orcid.org/0000-0001-8577-3798

Takahiro Matsuda

Hokkaido University

Wei Cui

Hokkaido University

Hinako Kato

Hokkaido University

Shotaro Namiki

Hokkaido University

Tomoko Yamazaki

Hokkaido University

Martin Frauenlob

Hokkaido University

Daniel King

Hokkaido University https://orcid.org/0000-0002-1377-5556

Takayuki Nonoyama

Hokkaido University https://orcid.org/0000-0001-8554-0636

Masumi Tsuda

Hokkaido University

Shinya Tanaka

Hokkaido University

Tasuku Nakajima

Hokkaido University https://orcid.org/0000-0002-2235-3478

Jian Ping Gong ( $\nabla$ gong@sci.hokudai.ac.jp )

Hokkaido University https://orcid.org/0000-0003-2228-2750 
Keywords:

Posted Date: February 22nd, 2022

DOI: https://doi.org/10.21203/rs.3.rs-1286903/v1

License: (c) (1) This work is licensed under a Creative Commons Attribution 4.0 International License. Read Full License 


\section{Instantaneous micropatterning of a double-network hydrogel \\ 2 surface triggered by mechanical force}

3 Qifeng $\mathrm{Mu}^{1}$, Kunpeng Cui ${ }^{2}$, Zhijian Wang ${ }^{1}$, Takahiro Matsuda ${ }^{3}$, Wei Cui ${ }^{3}$, Hinako Kato ${ }^{1}$, Shotaro Namiki ${ }^{1}$, 4 Tomoko Yamazaki ${ }^{1}$, Martin Frauenlob ${ }^{1}$, Daniel R. King ${ }^{3}$, Takayuki Nonoyama ${ }^{3}$, Masumi Tsuda ${ }^{2,4}$, Shinya

5 Tanaka ${ }^{2,4}$, Tasuku Nakajima ${ }^{2,3 *}$, Jian Ping Gong ${ }^{2,3 *}$

$6{ }^{1}$ Graduate School of Life Science, Hokkaido University, N21W11, Kita-ku, Sapporo 001-

7 0021, Japan;

8 Institute for Chemical Reaction Design and Discovery (WPI-ICReDD), Hokkaido University,

9 N21W10, Kita-ku, Sapporo 001-0021, Japan;

10 35aculty of Advanced Life Science, Hokkaido University, N21W11, Kita-ku, Sapporo 00111 0021, Japan;

12 4Department of Cancer Pathology, Faculty of Medicine, Hokkaido University, N15, W7, 13 Kita-ku, Sapporo 060-8638, Japan

14 Instantaneous patterning on soft material surfaces, such as hydrogels, is 15 important in various research fields; however, it is extremely challenging. 16 Existing strategies for soft material surface patterning are time-consuming and 17 have poor chemical versatility. Herein, we show how micropatterns can be rapidly created on tough double-network (DN) hydrogel surfaces via sacrificial bonds and mechanical force-triggered radical polymerisation. Micropatterning is performed by local mechanical forces, which initiate radical polymerisation in

21 a process zone in the presence of unsaturated monomers, resulting in on22 demand functional patterns with a variety of geometries and chemistries. Such force-triggered activation occurs within seconds and is applicable to diverse 
monomers. To demonstrate potential applications, we engineered topographies and chemistries on DN hydrogel surfaces to control cell adhesion and water droplet directional transport.

Soft materials with patterned surfaces have broad application scope ranging from adhesion and tissue engineering to actuators and flexible displays ${ }^{1-3}$. These patterns are not limited to shape but include the surface distribution of physical and chemical properties, such as stiffness and chemical species.

Several creative approaches have been demonstrated for creating patterns on soft materials ${ }^{4,5}$, particularly photolithography ${ }^{6,7}$, buckling instabilities ${ }^{8-10}$, printing ${ }^{11,12}$, and moulding ${ }^{13,14}$. Photolithography is one of the most common methods for the chemical patterning of soft material surfaces owing to its high resolution and possibility of on-demand patterning. However, during photolithography, reactions can occur only in the presence of appropriate photo-initiators or photoactive substrates. Mechanics-based strategies resulting in buckling instabilities have also been leveraged to form micropatterns on soft and elastic surfaces. However, this method is applicable only for a very limited number of shapes, such as wrinkles and arrays; additionally, it is not suitable for most functional polymers because appropriate material stiffness is required for the formation of the desired pattern. Moulding and assembling are chemistry-diverse strategies for forming various shapes on soft surfaces. However, these methods can only be used to form microstructures with the same type of material as the substrate and are not 
suitable for on-demand customisation of microstructures because moulding requires an expensive solid mould. Microcontact printing and three-dimensional (3D) printing have been recently developed for the formation of the desired surface structures; however, these methods require complex operations and expensive instruments. In addition to these shortcomings, these pioneering methods are time-consuming. For example, photolithography typically involves complicated processes (e.g. mask preparation and post-curing) that can range from tens of seconds to several hours. Currently, the development of an instantaneous strategy, which would be generally applicable regardless of the substrate or feature chemistry, while enabling precise and facile control of the microstructure topography and spatial presentation, is a considerable challenge. Herein, we present an instantaneous, simple, and chemistry-diverse strategy to create microstructures or patterns on the surfaces of DN hydrogels. DN hydrogels comprise two interpenetrating networks with contrasting structures and mechanical properties. The first network, highly crosslinked and prestretched, acts as a rigid but brittle skeleton on the molecular scale and determines the elastic modulus of DN hydrogels. The second network, sparsely crosslinked and relatively concentrated, is soft and stretchable ${ }^{15}$. In principle, DN hydrogels are chemistry-diverse and can be constructed from polymers of any chemical structure as long as the contrasting interpenetrating network structures are formed ${ }^{16}$. Owing to such contrasting structures, a large number of brittle first network strands can break during deformation without causing 
catastrophic failure of the hydrogel because the second network carries load once the strands in the first network break, which redistributes stress to other first network strands around the broken strands ${ }^{17-19}$. The fracture of the strands in the first brittle network typically occurs because of the homolytic scission of covalent bonds, which generates mechanoradicals at the broken ends of polymer strands ${ }^{20,21}$. The concentration of mechanoradicals is sufficiently high to initiate polymerisation inside DN hydrogels fed with monomers, which enables force-triggered growth of $\mathrm{DN}$ hydrogels in the presence of reactive monomers and crosslinkers. Monomers and crosslinkers act as growth-building elements, analogous to nutrients in the natural growth of living organisms. The newly synthesised polymer strands enable novel mechanical properties or functions of DN hydrogels. For example, muscle-like mechanical growth and increase in the thermo-responsivity of DN hydrogels have been achieved using this method ${ }^{22}$. As radical polymerisation is applicable to various functional unsaturated monomers, force-triggered polymerisation in DN hydrogels can, in principle, impart various chemical functions to the gels.

In our previous work, the mechanical force was typically applied uniformly to the DN hydrogel so that the polymerisation proceeded homogeneously inside the bulk ${ }^{22}$. In the present work, we applied local mechanical indentation on DN hydrogel surfaces under a monomer aqueous solution, which induced regioselective polymerisation of monomers in the DN hydrogels. Polymerisation occurred within tens of seconds, resulting in the nearly instantaneous formation 
of various physical and/or chemical microstructures on the surface layer of DN hydrogels (Fig. 1). In addition, we demonstrated novel functions of the patterned DN hydrogels, including highly stretchable tough display devices, cell orientation along the patterned structures, and directional liquid droplet transport. As this molecular-scale productive mechanochemistry is not limited to water-rich hydrogels ( 90 wt $\%$ of water) but could be applied to different types of multiple-network polymeric materials, including organogels and solvent-free elastomers ${ }^{23,24}$, it has the potential to dramatically impact the field of soft material surfaces and interfaces.

Controlling the DN hydrogel surface. We used DN hydrogels consisting of an anionic poly(2-acrylamido-2-methylpropanesulfonic acid sodium salt) (PNaAMPS) first network and nonionic polyacrylamide (PAAm) second network. The DN hydrogels were prepared by a two-step sequential polymerisation. Formation of surface microstructures on DN hydrogels through force-triggered radical polymerisation requires the use of DN hydrogels with a double-network structure on the top-most surface layer. It has been shown that DN hydrogels comprising an anionic first network prepared on glass moulds are covered by a nonionic second network layer several micrometres thick, because an electric double layer is formed at the interface between the anionic first network and glass, which are both negatively charged in water during the preparation of the second network ${ }^{25}$. To prevent the formation of the electric double layer, the glass mould surface was covered with a hydrophobic film during the synthesis 
of the second network (Fig. S1). The chemical signal of the first network on the DN hydrogel surface was detected by attenuated total reflectance Fouriertransform infrared spectroscopy (Fig. S2). The highly crosslinked and prestretched first network was confirmed to dominate the large surface elastic modulus of the DN hydrogels (Fig. S3, Table S1). These data suggest that the DN structure formed not only in the gel bulk but also near the gel surface without the formation of a soft surface layer from the second network. The mean roughness $\left(R_{a}\right)$ of the DN hydrogel surfaces, measured by 3D laser microscopy, was $\sim 0.9 \mu \mathrm{m}$ (Fig. S4).

Regioselective internal rupture. The surface of the prepared DN hydrogel was locally pressed using a cylindrical steel indenter with a diameter $d=568$ $\mu \mathrm{m}$ and displacement $L_{\max }=1000 \mu \mathrm{m}$ without feeding any monomers in air. Upon mechanical indentation, the DN hydrogel effectively dissipated energy, as

125 indicated by the pronounced mechanical hysteresis (Fig. S5). The dissipated 126 mechanical energy was considered to be used for homolytic cleavage of the 127 first brittle network strands in the pressed region. The cleavage of the first network appeared as a reswelling of the pressed region when the sample was

129 re-immersed in deionised water, which occurred because the constraint to the 130 second network by the pre-stretched first network was released by the rupture 131 of the first network ${ }^{26}$. The reswollen region had a maximum height of $\sim 20 \mu \mathrm{m}$ 132 and a diameter of $\sim 560 \mu \mathrm{m}$ (Fig. S6). These results confirmed that covalent 133 bonds ruptured locally near the mechanical indention tip, which produced 
mechanoradicals in this localised process zone.

We quantified the hysteresis energy $U_{\text {hys }}$ and maximum force $F_{\max }$ of the DN hydrogel surface as a function of the indentation displacement $L_{\max }$ (Fig. S8). Because an excessively large $L_{\max }(>1000 \mu \mathrm{m})$ induced surface microcracks, $L_{\max }$ was controlled in the range of $0-1000 \mu \mathrm{m}$ to avoid the formation of microcracks (Fig. S7). Uhys was estimated from $U_{\text {hys }}=\int_{0}^{L_{\max }}\left(F_{\text {load }}-F_{\text {unload }}\right) \mathrm{d} L$, where $F_{\text {load }}$ and $F_{\text {unload }}$ are the mechanical force values of the indentation loading and unloading curves, respectively. At small $L_{\max }(<500 \mu \mathrm{m})$, there was

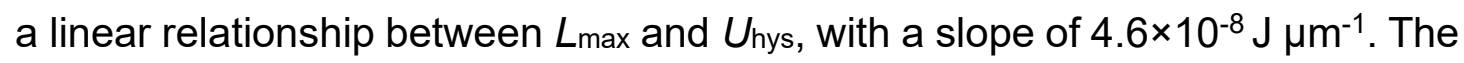
DN hydrogel surface underwent larger deformation at $L_{\max }$ above $500 \mu \mathrm{m}$, where the energy dissipation increased sharply. The amount of mechanical hysteresis $U_{\text {hys }}$ is considered to be proportional to the number of ruptured first network strands in the process zone. Assuming a process zone volume of $\pi(d / 2)^{2} L_{\max }$, a rough estimate of the mechanoradical concentration increases from 0 to $2.9 \times 10^{-5} \mathrm{M}$ as $L_{\max }$ increases from 0 to $1000 \mu \mathrm{m}$, according to our previous work ${ }^{22}$ (Fig. S8). In contrast to the energy dissipation of the DN hydrogels, the nearly elastic PAAm single-network (SN) hydrogel exhibited negligible mechanical hysteresis upon cyclic indentation (Fig. S9).

Micropatterning and properties. To realise surface micropatterning through mechanical force-triggered polymerisation, DN hydrogels were first immersed in various monomer aqueous solutions (Fig. 2a, b). Without any further treatment, these chemically crosslinked DN hydrogels were permeable to small 
molecules, enabling efficient radical polymerisation in the pressed regions of

157 the gel surface. The micropatterning of the gel surface triggered by a

158 regioselective mechanical force was carried out in a homemade experimental

159 setup under an argon atmosphere (Movie S1). After the indentation followed by

160 swelling, a convex micropattern was formed on the DN hydrogel surface. To

161 investigate the formed pattern, the pressed DN hydrogels were immersed in

162 deionised water, and the height $(H)$ of the prepared micropattern was

163 determined. The $H$ of the DN hydrogels fed with monomers was much higher

164 than the height observed in the reswollen sample (the indented DN hydrogel

165 not fed with monomer). For example, if the gel was fed with $\mathrm{N}$ -

166 isopropylacrylamide (NIPAm), the $H$ of the microstructure was $\sim 60 \mu \mathrm{m}$, which

167 is much higher than the height observed in the reswollen sample $(\sim 20 \mu \mathrm{m})$ for

168 the same indenter $d=568 \mu \mathrm{m}$ at $L_{\max }=1000 \mu \mathrm{m}$ (Fig. 2c). Using selective

169 mechanical indentation, we monitored the patterning and properties of

170 microstructures in well-defined process zones. The height of the

171 microstructures could be regulated by the mechanical hysteresis energy, as

172 revealed by their almost proportional relationship (Fig. 2d, e). The diameters of

173 the microstructures were almost the same as that of the indenter (Fig. $2 \mathrm{f}$ and

174 Fig. S10, 11). Therefore, the topographic height, diameter, and shape of the

175 microstructures can be easily regulated based on the application requirements.

176 As force-triggered radical polymerisation is generic, we further used a series of

177 monomers to fabricate various microstructures on DN hydrogel surface. The 
typical profiles, topographic height, and diameter of the microstructures were

compared. The anionic monomer NaAMPS showed the highest topographic height among all the evaluated micropatterning systems. There was an obvious dependence between the topographic height and monomer species (Fig. $2 \mathrm{~g}$ and Fig.S12).

To elucidate the underlying mechanisms of micropatterning using different monomers, we further investigated the monomer conversion ratio in forcetriggered radical polymerisation. In this study, stretched DN hydrogels with monomers were characterised. The results reveal that the conversion ratio of NaAMPS $(\sim 0.80)$ is the highest, and the conversion ratio of NIPAm $(\sim 0.69)$ is higher than those of the other three ionic monomers (AAc, NaSS, and MPTC) in the course of the force-triggered radical polymerisation (Fig. 2h and Fig. S13, 14). We propose that the conversion ratio is one of the main factors causing topographic height differences because a higher conversion ratio induces a higher osmotic pressure difference. The difference in the conversion ratio of these reactive monomers may be related to the substituent groups on the vinyl and/or acryloyl units, or the polarity of the monomer functional groups, which determines the relative stability of the $\mathrm{C}=\mathrm{C}$ bonds during radical polymerisation ${ }^{27,28}$. Furthermore, we showed that the presence of a crosslinker $\left(N, N^{\prime}\right.$-methylenebisacrylamide, MBA) does not affect both the conversion ratio and topographic height (Fig. S15-S17). The topographic height of the microstructures was largely dependent on the feed monomer concentration (Fig. 
$\mathrm{S} 16 \mathrm{~b}, 17 \mathrm{~b})$. In addition, the microstructures were stimuli-responsive to temperature and $\mathrm{pH}$ changes when formed from NIPAm and AAc, respectively (Fig. S18, S19).

Kinetics of force-triggered micropatterning. Nearly instantaneous micropatterning includes three main processes: bond scission, chain growth, and swelling. As demonstrated in Fig.3 and movie S2, force-triggered bond scission and radical polymerisation occur instantaneously, within tens of seconds. Bond scission during tension is essentially a thermally activated process, which may also be modelled as an ultrafast first-order kinetic process $^{29-31}$. The time scale $\bar{\tau}_{1}$ for bond scission is estimated as $10^{-12} \mathrm{~s}$ for a polymer strand that experiences a force of $\sim 6 \mathrm{nN}^{31}$. Force-triggered radical polymerisation occurs through molecule-by-molecule addition growth, which implies the sequential formation of individual bonds. The active centres (i.e.

213 chain-end mechanoradicals) induced by bond scission in the polymer

214 backbones are retained by a single polymer molecule throughout its growth.

215 The radicals can be quenched by the well-known chain-terminating process during radical polymerisation ${ }^{32}$. Time-resolved near-infrared spectroscopy

217 revealed that the force-triggered radical polymerisation was almost completed 218 within tens of seconds (Fig. S20); hence, the polymerisation timescale $\bar{\tau}_{2}$ was 219 estimated to be $10^{0}-10^{1} \mathrm{~s}$. After chain growth, the microstructures immediately 220 swell in an aqueous solution under osmotic pressure. The time scale of the 221 swelling is governed by the diffusion of the polymer network and water ${ }^{33,34}$. In 
our system, we found that the time scale $\bar{\tau}_{3}$ for swelling to equilibrium of a small process zone (thickness of $\sim 1000 \mu \mathrm{m}$ and diameter of $\sim 600 \mu \mathrm{m}$ ) was within 1 min (Fig. S21). Time-lapse microscope observation of the microstructure on DN hydrogel surface revealed that the instantaneous micropatterning was finished earlier than 4 minutes (Fig. S22). We propose that the nearly instantaneous micropatterning is a force and temperature-dependent dynamic process, which is dominated by the above-mentioned three processes. Despite micropatterning occurring through a sequential, multi-step process, the overall rate of micropatterning is determined by the kinetics of the slowest step, i.e. swelling.

Programmable patterning. The proposed mechanochemical fabrication strategy for the formation of micropatterns is applicable not only to simple convex patterns but also to more complex patterns on a larger scale. We prepared a series of complex patterned indenters using a precisely controlled 3D printing system (Fig. S23, S24). We created various thermo-responsive poly ( $N$-isopropylacrylamide) (PNIPAm) patterns on gel surfaces, and used a fluorescent molecular probe, 8-anilino-1-naphthalenesulfonic acid (ANS), which emits a strong fluorescence signal in hydrophobic environments, to image the patterns (Fig. S25) ${ }^{35,36}$. Fig. 4a(i-v) shows various fluorescent patterns on DN hydrogel surfaces prepared using dynamic and programmable patterning. In addition, a bamboo-knot-like pattern (Fig. 4a(vi)) and parallel stripe pattern (Fig. $4 a(v i i))$ in the gel bulk were also prepared using regioselective mechanical indentation. In response to temperature and ultraviolet light (UV) stimuli, a 
fluorescent pattern that reads "Hello" was reversibly cycled on/off over multiple cycles (Fig. 4b and Movie S3). The patterned DN hydrogel with excellent mechanical properties can potentially be used in applications such as stretchable and tough display devices (Fig. 4c).

Specific applications of micropatterned DN hydrogels. To illustrate the potential applications of this nearly instantaneous surface patterning strategy, we prepared a DN hydrogel surface with parallel PNIPAm patterns (Fig. 5a) using a stacked metal blade (Fig. S26). First, we demonstrated the programmed cell orientation on the PNIPAm-patterned DN hydrogel surface. The myoblast cells (C2C12) labelled with a green fluorescent protein (GFP) were cultured on the DN hydrogel surface at $37{ }^{\circ} \mathrm{C}$, which is higher than the lower critical solution temperature of PNIPAm. The aligned hydrophobic PNIPAm domains on the DN hydrogels induced cell aggregation and orientation through interactions with cells. Contact guidance was derived from an orchestrated interplay between the extracellular matrix, biophysical cues, and cell recognition sites that affect their decision ${ }^{37}$. The fluorescence microscopy images showed clear differences in cellular orientation across the patterned and non-patterned DN hydrogel surfaces (Fig. 5b). Some inconspicuous alignments were observed within the micropatterned constructs on day 1 of culturing. By day 5 , the cells filled the microstructures and were mostly aligned along the major axis of the orientation pattern (Fig. $5 b(i i))$. In contrast to the micropatterned regions, the cell nuclei were randomly oriented within the non- 
patterned regions (Fig. $5 b(v))$. To assess the role of microstructures in cellular alignment, we quantified the $\mathrm{C} 2 \mathrm{C} 12$ nuclear alignment within the patterned and non-patterned regions after 5 days of culturing. Cell nuclear alignment was grouped in $10^{\circ}$ increments to compare it with the alignment distribution under different conditions (Fig. 5b(vi)).

Next, we showed how anisotropic DN hydrogel surfaces with parallel PNIPAm patterns can be used for water droplet directional transport. When placed on DN hydrogels patterned with parallel PNIPAm lines, water droplet morphology is dependent on the surface pattern structures (Fig. 5c), which indicates that the patterns can be used to regulate surface wettability. In situ observation of

276 the water spreading and transportation process on the patterned hydrogel 277 surface showed that the water contact line expanded rapidly along with the 278 parallel patterns (Movie S4, S5). Here, the parallel patterns acted as an adaptable path that stabilised and induced water droplet sliding and expansion 280 along the pattern direction (Fig. S27-S30). In addition, there were many rough 281 microchannels on the surface of the parallel patterns (Fig. S26c), which was 282 beneficial for water wetting owing to the enhanced capillary force ${ }^{38}$. Once the external forces were out of balance, the water droplet moved in the direction of

284 the patterns. The vertically patterned surface induced faster water transport, 285 and the transport velocity was dependent on the water droplet volume (Fig. S31). 


\section{Conclusions}

The formation of microstructures or patterns through metabolic processes is frequently encountered in biological systems. Herein, we report a regioselective mechanochemical strategy for nearly instantaneous micropatterning on tough DN hydrogel surfaces using sacrificial bonds and mechanical force-triggered radical polymerisation. The demonstrated method of rapid micropatterning of soft material surfaces enables a number of future research directions and applications. We envision that our instantaneous micropatterning technique holds promise for microsensor arrays, soft adhesion, flexible displays, and biomedical devices. Our studies with well-defined artificial polymer systems might also contribute to the expansion of a rather limited understanding of mechanochemical transduction in biological systems.

\section{References}

1. Bartlett, M. D. et al. Looking beyond fibrillar features to scale gecko-like adhesion. Adv. Mater. 24, 1078-1083 (2012).

2. $\mathrm{Wu}, \mathrm{Z}$. L. et al. Three-dimensional shape transformations of hydrogel sheets induced by small-scale modulation of internal stresses. Nat. Commun. 4, 1-7 (2013).

3. Wang, Q. M., Gossweiler, G. R., Craig, S. L. \& Zhao, X. H. Cephalopod-inspired design of electro-mechano-chemically responsive elastomers for on-demand fluorescent patterning. Nat. Commun. 5, 1-9 (2014).

4. Nie, Z. H. \& Kumacheva, E. Patterning surfaces with functional polymers. Nat. Mater. 7, 277-290 (2008).

5. Munoz-Robles, B. G., Kopyeva, I. \& Deforest, C. A. Surface patterning of hydrogel biomaterials to probe and direct cell-matrix interactions. Adv. Mater. Interfaces 7 , 2001198 (2020).

6. Wang, Z. J. et al. Cooperative deformations of periodically patterned hydrogels. Sci. Adv. 3, e1700348 (2017). 
7. Xue, L. L. et al. Light-regulated growth from dynamic swollen substrates for making rough surfaces. Nat. Commun. 11, 1-9 (2020).

8. Kim, J., Yoon, J. \& Hayward, R. C. Dynamic display of biomolecular patterns through an elastic creasing instability of stimuli-responsive hydrogels. Nat. Mater. 9, 159-164 (2010).

9. Cao, C. Y. et al. Harnessing localized ridges for high-aspect-ratio hierarchical patterns with dynamic tunability and multifunctionality. Adv. Mater. 26, 1763-1770 (2014).

10. Ouchi, T., Yang, J. W., Suo, Z. G. \& Hayward, R. C. Effects of stiff film pattern geometry on surface buckling instabilities of elastic bilayers. ACS Appl. Mater. Interfaces 10, 23406-23413 (2018).

11. Kim, Y. et al. Printing ferromagnetic domains for untethered fast-transforming soft materials. Nature 558, 274-279 (2018).

12. Liu, X. J. et al. 3D printing of bioinspired liquid superrepellent structures. Adv. Mater. 30, 1800103 (2018).

13. Baik, S. et al. A wet-tolerant adhesive patch inspired by protuberances in suction cups of octopi. Nature 546, 396-400 (2017).

14. Rao, P. et al. Tough hydrogels with fast, strong, and reversible underwater adhesion based on a multiscale design. Adv. Mater. 30, 1801884 (2018).

15. Gong, J. P., Katsuyama, Y., Kurokawa, T. \& Osada, Y. Double-network hydrogels with extremely high mechanical strength. Adv. Mater. 15, 1155-1158 (2003).

16. Nakajima, T. et al. A universal molecular stent method to toughen any hydrogels based on double network concept. Adv. Funct. Mater. 22, 4426-4432 (2012).

17. Webber, R. E. et al. Large strain hysteresis and Mullins effect of tough double-network hydrogels. Macromolecules 40, 2919-2927 (2007).

18. Yu, Q. M. et al. Direct observation of damage zone around crack tips in double-network gels. Macromolecules 42, 3852-3855 (2009).

19. Long, R., Hui, C. Y., Gong, J. P. \& Bouchbinder, E. The fracture of highly deformable soft materials: a tale of two length scales. Annu. Rev. Condens. Matter. Phys. 12, $71-$ 94 (2021).

20. Simon, Y. C. \& Craig, S. L. Mechanochemistry in Materials Ch. 2 (Royal Society of Chemistry, 2017).

21. Sohma, J. Mechanochemistry of polymers. Prog. Polym. Sci. 14, 451-596 (1989).

22. Matsuda, T. et al. Mechanoresponsive self-growing hydrogels inspired by muscle training. Science 363, 504-508 (2019).

23. Ducrot, E. et al. Toughening elastomers with sacrificial bonds and watching them break. 
Science 344, 186-189 (2014).

24. Matsuda, T. et al. Fabrication of tough and stretchable hybrid double-network elastomers using ionic dissociation of polyelectrolyte in nonaqueous media. Chem. Mater. 31, 3766-3776 (2019).

25. Frauenlob, M. et al. Modulation and characterization of the double network hydrogel surface-bulk transition. Macromolecules 52, 6704-6713 (2019).

26. Nakajima, T. et al. Characterization of internal fracture process of double network hydrogels under uniaxial elongation. Soft Matter 9, 1955-1966 (2013).

27. Zubov, V. P., Kumar, M. V., Masterova, M. N. \& Kabanov, V. A. Reactivity of allyl monomers in radical polymerization. J. Macromol. Sci. Chem. 13, 111-131 (1979).

28. Matyjaszewski, K. \& Davis, T. P. Handbook of Radical Polymerization Ch. 1 (John Wiley \& Sons. Press, 2003).

29. Zhurkov, S. N. Kinetic concept of the strength of solids. Int. J. Fract. Mech. 1, 311-323 (1965).

30. Horn, A. F. \& Merrill, E. W. Midpoint scission of macromolecules in dilute solution in turbulent flow. Nature 312, 140-141 (1984).

31. Beyer, M. K. The mechanical strength of a covalent bond calculated by density functional theory. J. Chem. Phys. 112, 7307-7312 (2000).

32. Hiemenz, P. C. \& Lodge, T. P. Polymer Chemistry Ch. 3 (CRC. Press, 2007).

33. Tanaka, T. \& Fillmore, D. J. Kinetics of swelling of gels. J. Chem. Phys. 70, 1214-1218 (1979).

34. Fujiyabu, T. et al. Temperature dependence of polymer network diffusion. Phys. Rev. Lett. 127, 237801 (2021).

35. $\mathrm{Mu}, \mathrm{Q}$. F. et al. Robust multiscale-oriented thermoresponsive fibrous hydrogels with rapid self-recovery and ultrafast response underwater. ACS Appl. Mater. Interfaces 12, 33152-33162 (2020).

36. Matsuda, T., Kawakami, R., Nakajima, T. \& Gong, J. P. Crack tip field of a doublenetwork gel: visualization of covalent bond scission through mechanoradical polymerization. Macromolecules 53, 8787-8795 (2020).

37. Bettinger, C. J., Langer, R. \& Borenstein, J. T. Engineering substrate topography at the micro- and nanoscale to control cell function. Angew. Chem. Int. Edit. 48, 54065415 (2009).

38. Chen, H. W. et al. Continuous directional water transport on the peristome surface of Nepenthes alata. Nature 532, 85-89 (2016). 


\section{Online Methods}

384 Preparation of DN hydrogels with controlled surfaces. We adopted the previously-developed surface-bulk transition technology 23 to prepare DN hydrogels with no soft second network surface layer. First, the synthesised PNaAMPS SN hydrogel (monomer concentration $C_{1}$ of $1.0 \mathrm{M}, 4 \mathrm{~mol} \%$ crosslinker MBA, and $1 \mathrm{~mol} \%$ 2-oxoglutaric acid, molar ratio with respect to NaAMPS monomer) was immersed in the precursor aqueous solution of the PAAm second network (monomer concentration $C_{2}$ of $2.0 \mathrm{M}, 0.01 \mathrm{~mol} \% \mathrm{MBA}$, and $0.01 \mathrm{~mol} \%$ 2-oxoglutaric acid, molar ratio with respect to AAm monomer) for $1 \mathrm{~d}$. Then, the immersed PNaAMPS SN hydrogel was sandwiched between two flat plates to obtain a reaction cell. The plates were made of glass covered with a silicone-coated PET film. The reaction cell was compressed at a pressure of $\sim 0.8 \mathrm{kPa}$ for $12 \mathrm{~h}$ using the weight of several glass plates. Using this method, contact of the PNaAMPS first network with the hydrophobic surface of the cell wall was ensured. The reaction cell was then irradiated with UV light (365 nm, $4 \mathrm{~mW} \mathrm{~cm}^{-2}$ ) for at least $8 \mathrm{~h}$ in an argon glove box to synthesise the PAAm network in the presence of the first pre-stretched rigid PNaAMPS network. For reference, PAAm SN gel was also synthesised from an aqueous solution of 2.0 M AAm, $0.01 \mathrm{~mol} \% \mathrm{MBA}$, and $0.01 \mathrm{~mol} \%$ 2-oxoglutaric acid. The synthesised DN hydrogels and reference PNaAMPS and PAAm SN hydrogels were immersed in deionised water for later use.

Micropatterning of DN hydrogel surfaces. Ultrafast micropatterning was performed in an argon glove box using a mechanical tester (MCT-2150, A\&D Co., Ltd.). First, the cut DN hydrogels were immersed in deionised water to quench the mechanoradicals around the damaged cutting edges for $12 \mathrm{~h}$. The quenched DN hydrogels were immersed in a solution of different reactive monomers and crosslinkers overnight to incorporate the nutrients for subsequent patterning. Then, every DN hydrogel containing monomers and crosslinkers was moved to an argon glove box to remove oxygen. The DN hydrogels were fixed on a rigid glass plate with glue and then selectively indented in the solution using a cylindrical steel indenter, which was fixed on a small tensile machine using a screw. After the indentation, the gel was left still in the monomer solution for approximately $4 \mathrm{~min}$, then it was picked up from the glove box. Finally, the hydrogels were immersed in deionised water for at least 1 min to obtain swollen microstructures on the hydrogel surfaces. The complex 
patterns on the DN hydrogel surfaces were prepared using 3D printed resin indenters. The parallel patterns were prepared using stacked metal blades, the distance between which was controlled using silicone rubber spacers (thickness of $\sim 500 \mu \mathrm{m}$ ).

Cell adhesion to patterned DN hydrogel surfaces. The mouse myoblast cell line C2C12, labelled with GFP, was kindly provided by S. Kohsaka. The cells were cultured in Dulbecco's modified Eagle's medium containing $10 \%$ foetal bovine serum and $1 \%$ penicillin/streptomycin at $37{ }^{\circ} \mathrm{C}$ in a humidified atmosphere containing $5 \% \mathrm{CO}_{2}$. The $4-7$ th passages of the $\mathrm{C} 2 \mathrm{C} 12$ cells were used in this study. After sterilisation by UV light, the patterned DN hydrogel disk was placed in a 6-well polystyrene tissue culture dish, and the C2C12 cells were seeded on the patterned DN hydrogel surfaces at an initial cell density of $1 \times 10^{5} / \mathrm{mL}$. A cell dispersion solution was applied onto the hydrogel surface in a dropwise manner to produce a relatively uniform distribution of cells on the hydrogel surface. All cell-loaded hydrogel samples were cultured at $37^{\circ} \mathrm{C}$ in a humidified atmosphere containing $5 \% \mathrm{CO}_{2}$. The samples were transferred to new wells for observation under a microscope. The cell morphology and proliferation on the patterned DN hydrogel surface were monitored using an Olympus IX 71 fluorescence microscope (Olympus, Japan) equipped with a digital CCD camera with a lens (magnification of $\times 10$ ).

Water droplet directional transport. Droplets of deionised water of different volume $(2-25 \mu \mathrm{L})$ were used. The water droplets were dyed with methylene blue before use. The dyed water droplets were deposited using a micropipette. As soon as each droplet contacted the hydrogel surface, the droplet dynamics on the hydrogel surface was monitored using a commercially available camera. The relative humidity was maintained at approximately $30 \%$, and the room temperature was maintained at $25^{\circ} \mathrm{C}$.

Further details of these methods are available in the Supplementary Information.

\section{Data and materials availability}

The authors declare that data supporting the findings of this study are available within the paper and its supplementary information files.

\section{Acknowledgements}

We thank Y. Katsuyama for the preparation of the experimental devices and S. 
451 Kohsaka (National Cancer Center Research Institute, Tokyo, Japan) for 452 providing the C2C12-GFP cells. Funding: This research was supported by 453 JSPS KAKENHI grants JP17H06144, JP17J09290, and JP17H04891. Q. M. 454 thanks the China Scholarship Council (CSC no. 201808120092) for financial 455 support.

\section{Author contributions}

457 Q. M., K. C., T. N., and J. P. G. conceived the concept; Q. M., K. C., T. M., Z. 458 W., H. K., S. N., T. Y., M. F., M. T., and S. T. designed the experiments; Q. M., 459 Z. W., H. K., S. N., D. R. K., T. N., M. T., S. T., and T. N. performed the 460 experiments and analysed the data; Q. M., T. N., and J. P. G. wrote the 461 manuscript with the help from K. C., T. M., W. C., D. R. K., and M. T.

462 Competing interests

463 The authors declare no conflicts of interest. 


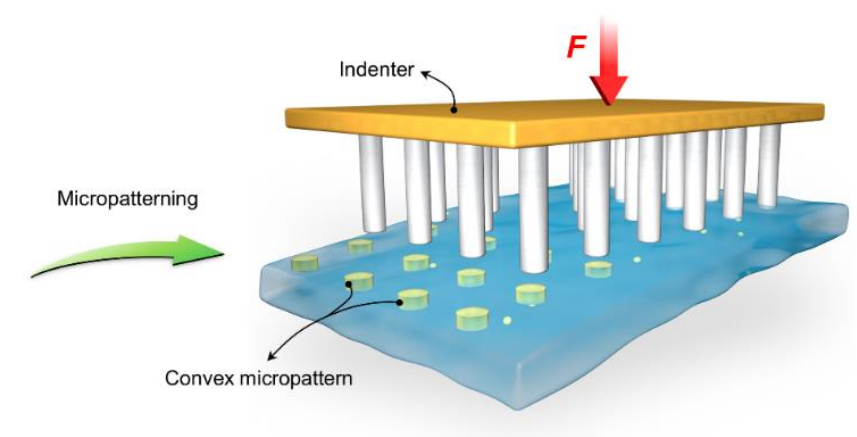

$\cdot$

i

Solution

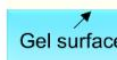

Gel surface
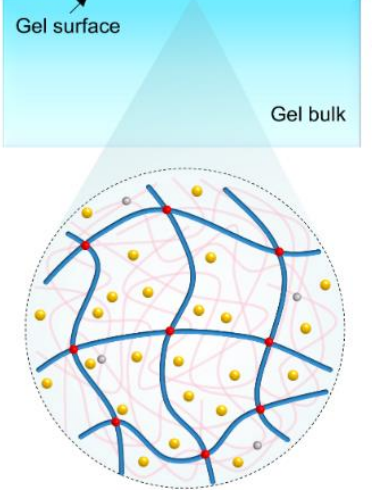

Double network
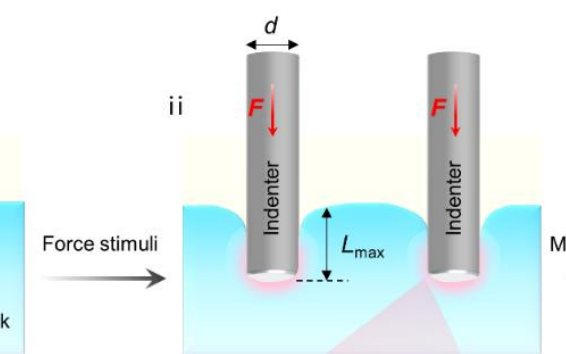

iii

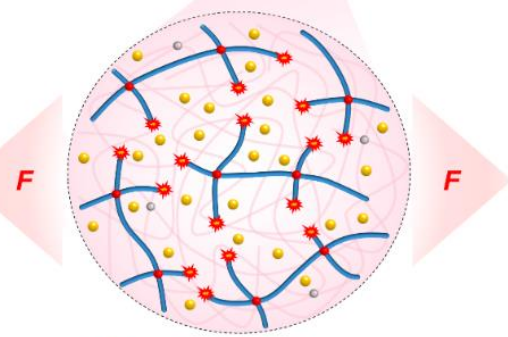

Chains scission in process zone

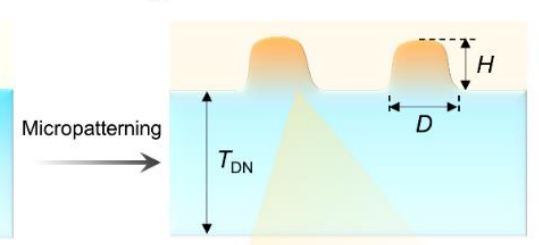

First network (brittle) $\Rightarrow$ Second network (stretchable) Monomer $\quad$ Mechanoradical Normed polymer chains

464

Fig.1|A mechanochemical strategy to develop instantaneous micropatterning on DN hydrogel surfaces. a, Schematic illustration for instantaneous micropatterning triggered by mechanical indentation under sufficient monomer supply. b. Molecular mechanism. The first rigid network is locally broken by compression from the cylindrical steel indenters, which generates mechanoradicals at the broken ends of the polymer strands in the process zone (pink region); with the presence of monomers, the mechanoradicals initiate fast growth of new polymer chains; the new polymer causes excess swelling, resulting in the formation of topological and chemical microstructures on the DN hydrogel surface. 
a

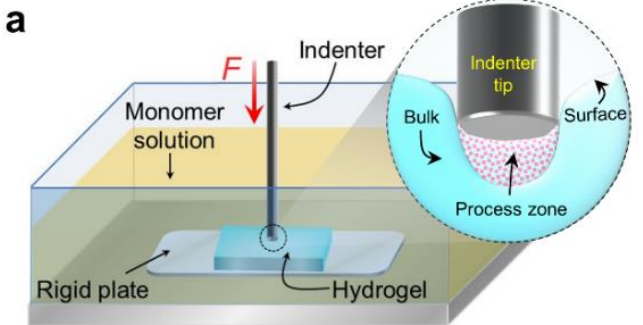

C
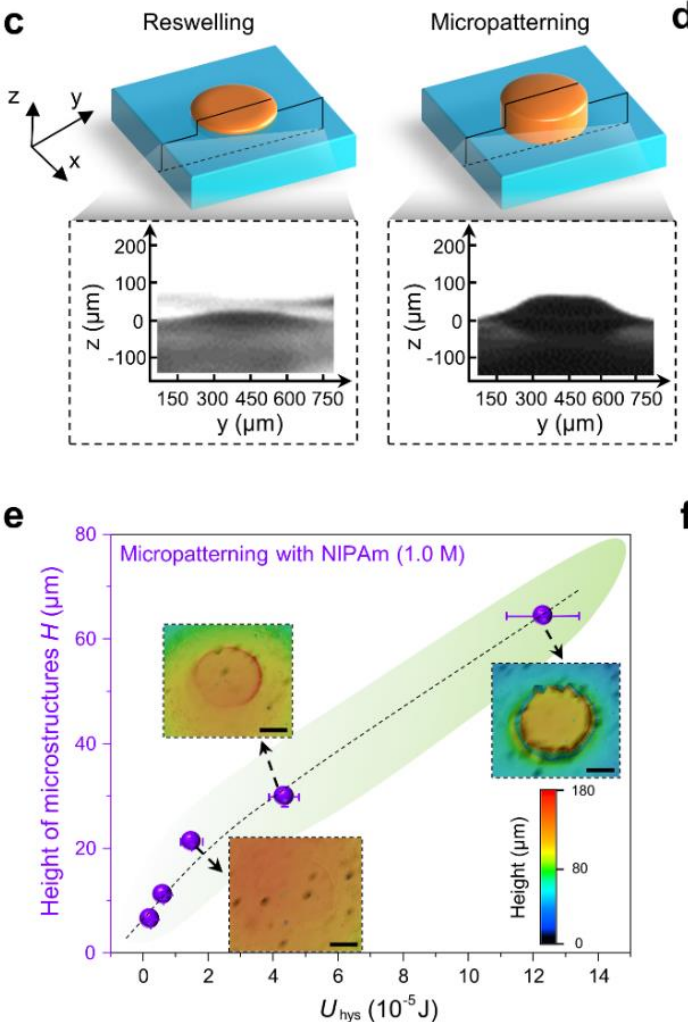

g

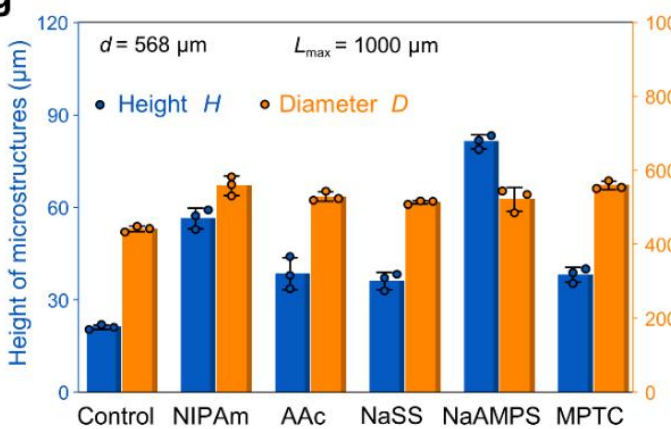

b Monomers

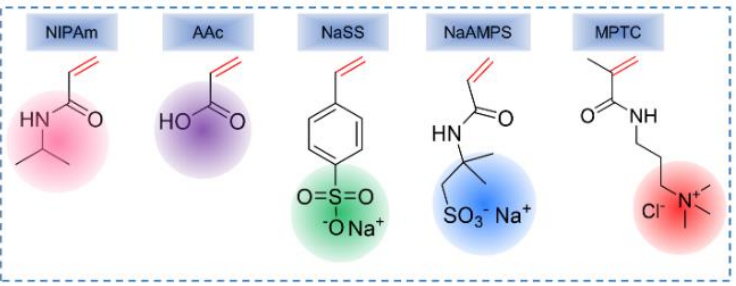

d

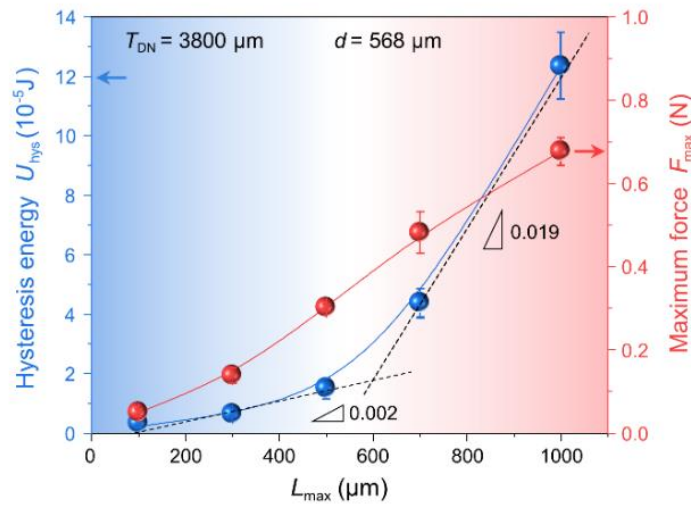

f

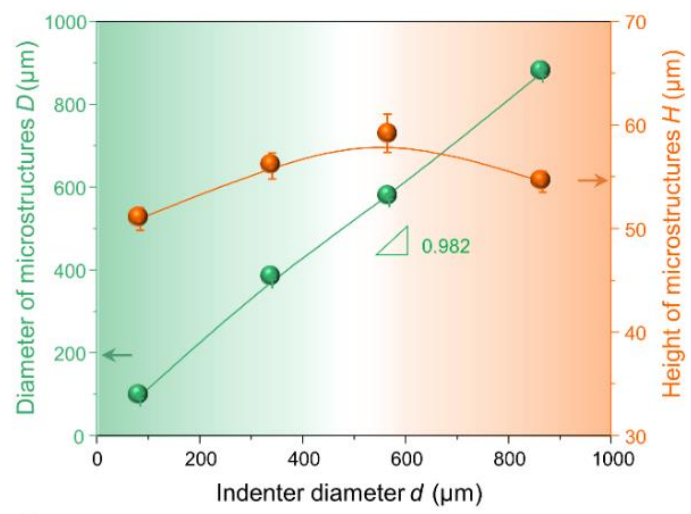

$\mathbf{h}$

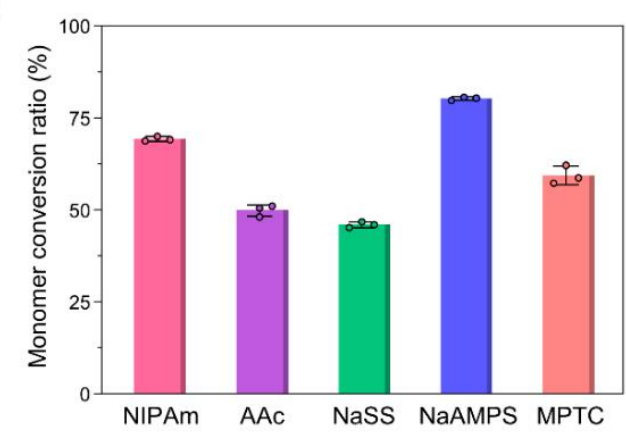

Fig.2| Mechanical force-regulated micropatterning with different monomers. a, Schematic of the experimental setup for mechanical indentation under monomer solution. Inset, the process zone around a cylindrical steel indenter with a flat tip on the DN hydrogel surface layer. b, Molecular structures of functional unsaturated monomers used for micropatterning, the different highlight colors represent different functional groups of monomers. c, Schematic illustration and representative microscope images (side view) of the DN hydrogel surface after compression by an indenter in the reswelling (left) and micropatterning (right) states. d, Dependences of hysteresis energy $U_{\text {hys }}$ and maximum force $F_{\max }$ in the loading-unloading curves on indentation displacements $L_{\max }$ for DN 
hydrogels of thickness $T_{\mathrm{DN}}=3800 \mu \mathrm{m}$ (DN gel fed with monomer NIPAm). e, Dependence of the topographical height of microstructures on the mechanical hysteresis energy $U_{\text {hys. }}$. Scale bar, $200 \mu \mathrm{m}$. f, Topographical diameter and height of microstructures versus indenter diameter $d$ for micropatterning with NIPAm on DN hydrogel surfaces $\left(L_{\max }=1000 \mu \mathrm{m}\right) . \mathbf{g}$, Topographical height and diameter of the microstructures using different monomers. $\mathbf{h}$, Conversion ratio of different functional unsaturated monomers in the mechanical forcetriggered radical polymerization, which was characterized in tensile specimens with different monomers stretched to a strain of $\varepsilon \sim 3$. The monomer concentration of $1.0 \mathrm{M}$ was used for $\mathrm{c}-\mathrm{h}$. Error bars represent the standard deviation from three replicates. The circular dots of the histogram in $\mathrm{g}$ and $\mathrm{h}$ represent the measured data points.

a Instantaneous patterning in NIPAm aqueous solution
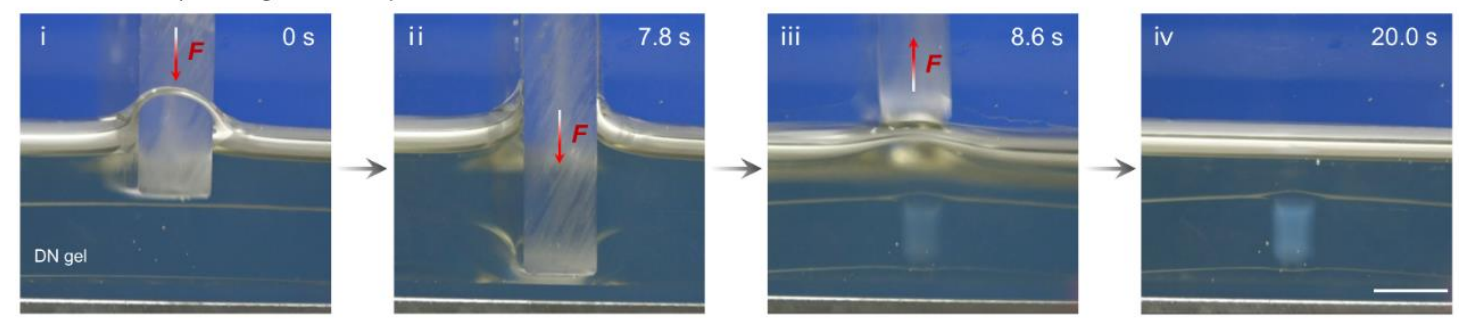

b Mechanical indentation in water (control)
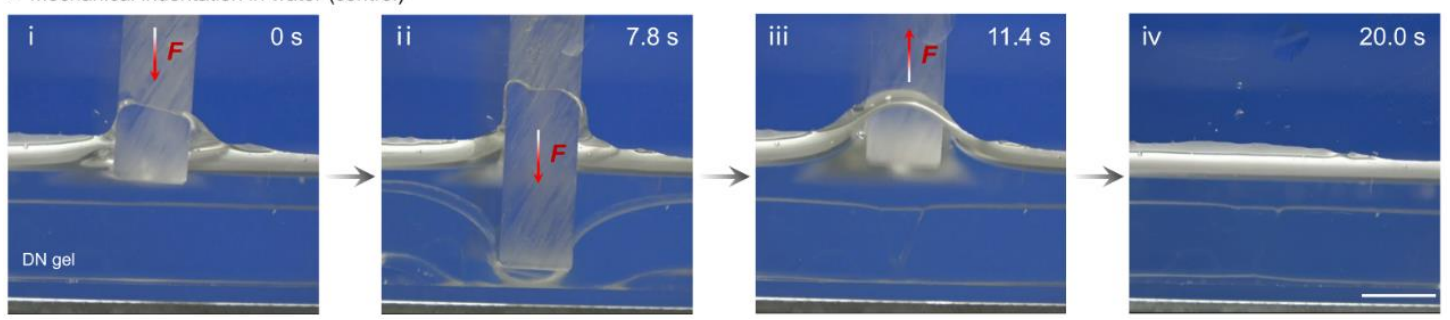

Fig.3| Time sequential snapshots to show instantaneous patterning triggered by mechanical force. a, Patterning in monomer aqueous solution (Here, concentration of NIPAm was $1.5 \mathrm{M}$ ). $\mathbf{b}$, In deionized water without a mechanochemically prepared patterns. Scale bars, $5 \mathrm{~mm}$. 

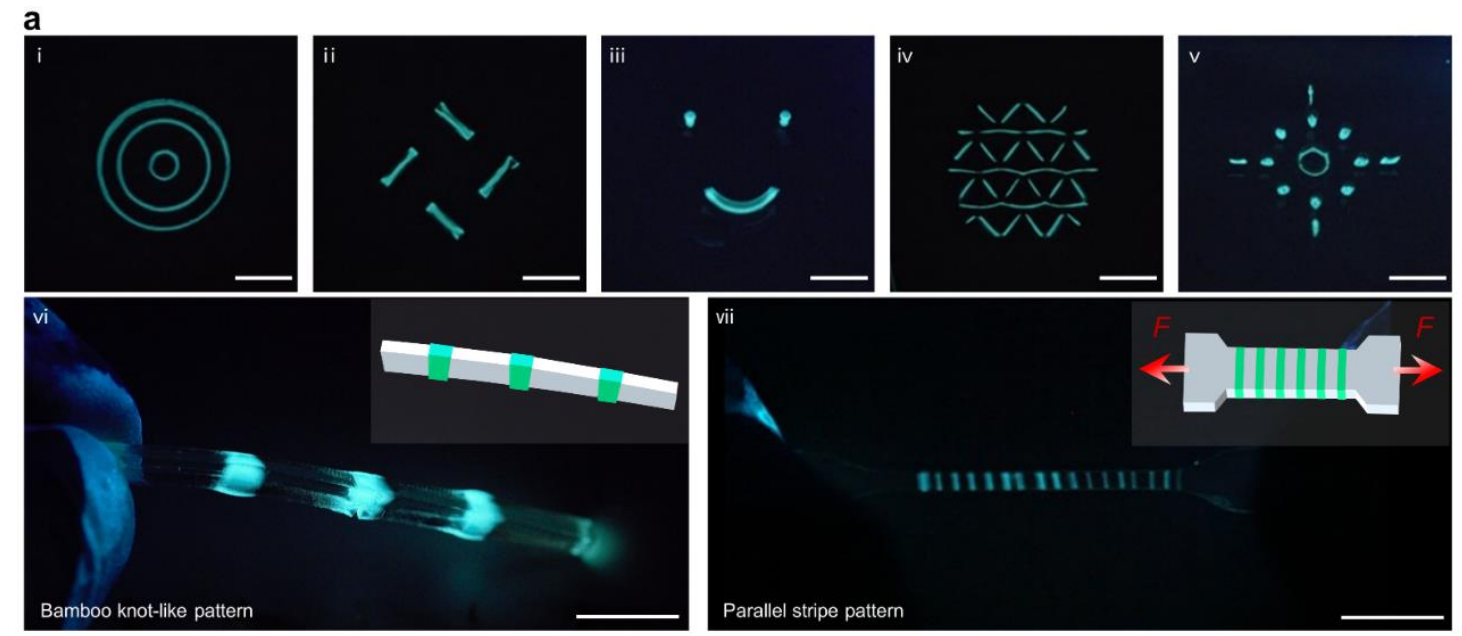

b On-off switching of fluorescence emission
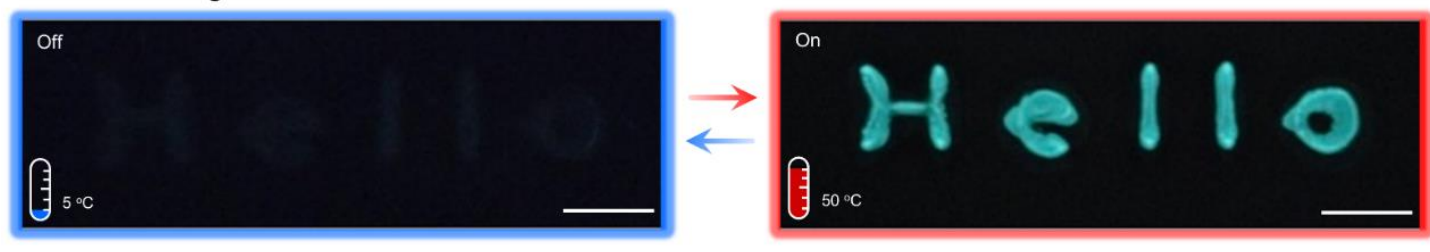

C Stretchable and tough display device
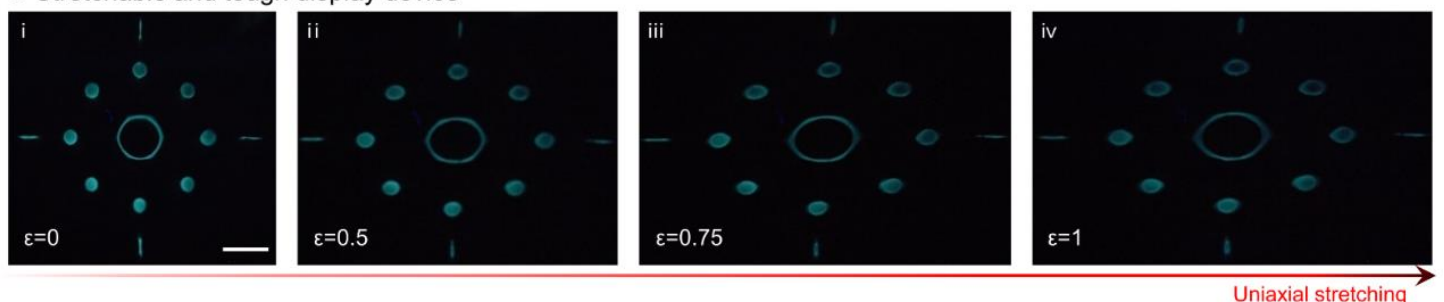

Fig.4| Programmable patterning of DN hydrogels. a, Fluorescence images showing various complex patterns on DN hydrogel surfaces (i-v). Scale bars, $5 \mathrm{~mm}$. Programmable patterns in the DN hydrogel bulk (vi, vii). Insets, cartoon representation of the bamboo knotlike pattern and parallel stripe pattern under stretching. Scale bars, $10 \mathrm{~mm}$. b, A fluorescent pattern that reads "Hello" for the stimulus-responsive display device. The left image highlighted with blue frame indicates the low temperature at $5^{\circ} \mathrm{C}$, and the right image with red frame indicates the high temperature at $50{ }^{\circ} \mathrm{C}$. The cyclic change of external temperature can trigger the on-off switching of fluorescence emission. Scale bars, $2.5 \mathrm{~mm}$. c, A patterned DN hydrogel was uniaxially stretched to different strains $\varepsilon$, which can act as a stretchable display device. NIPAm 1.0 M was used for a-c. Scale bar, $5 \mathrm{~mm}$. 

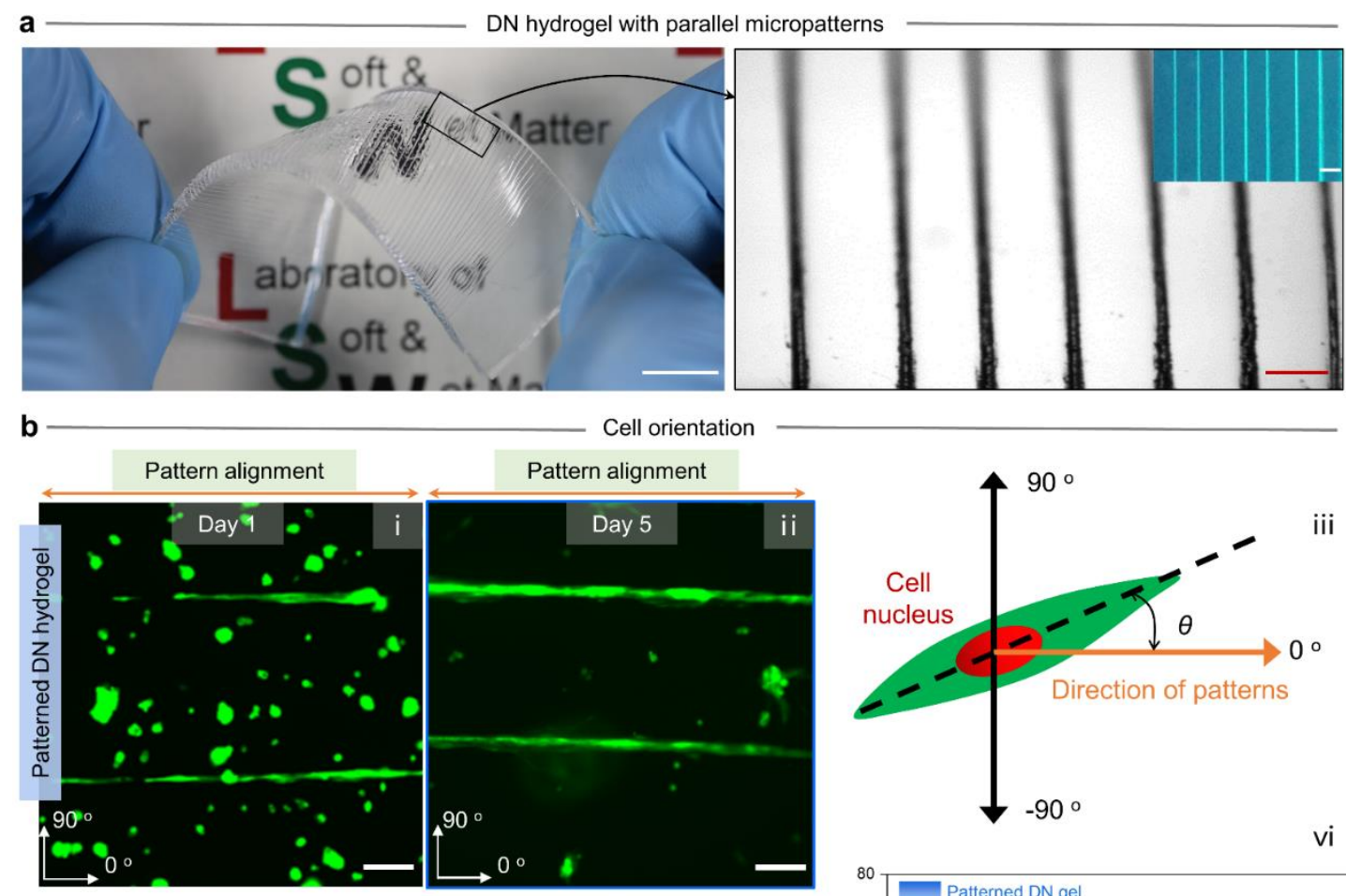

Cell orientation
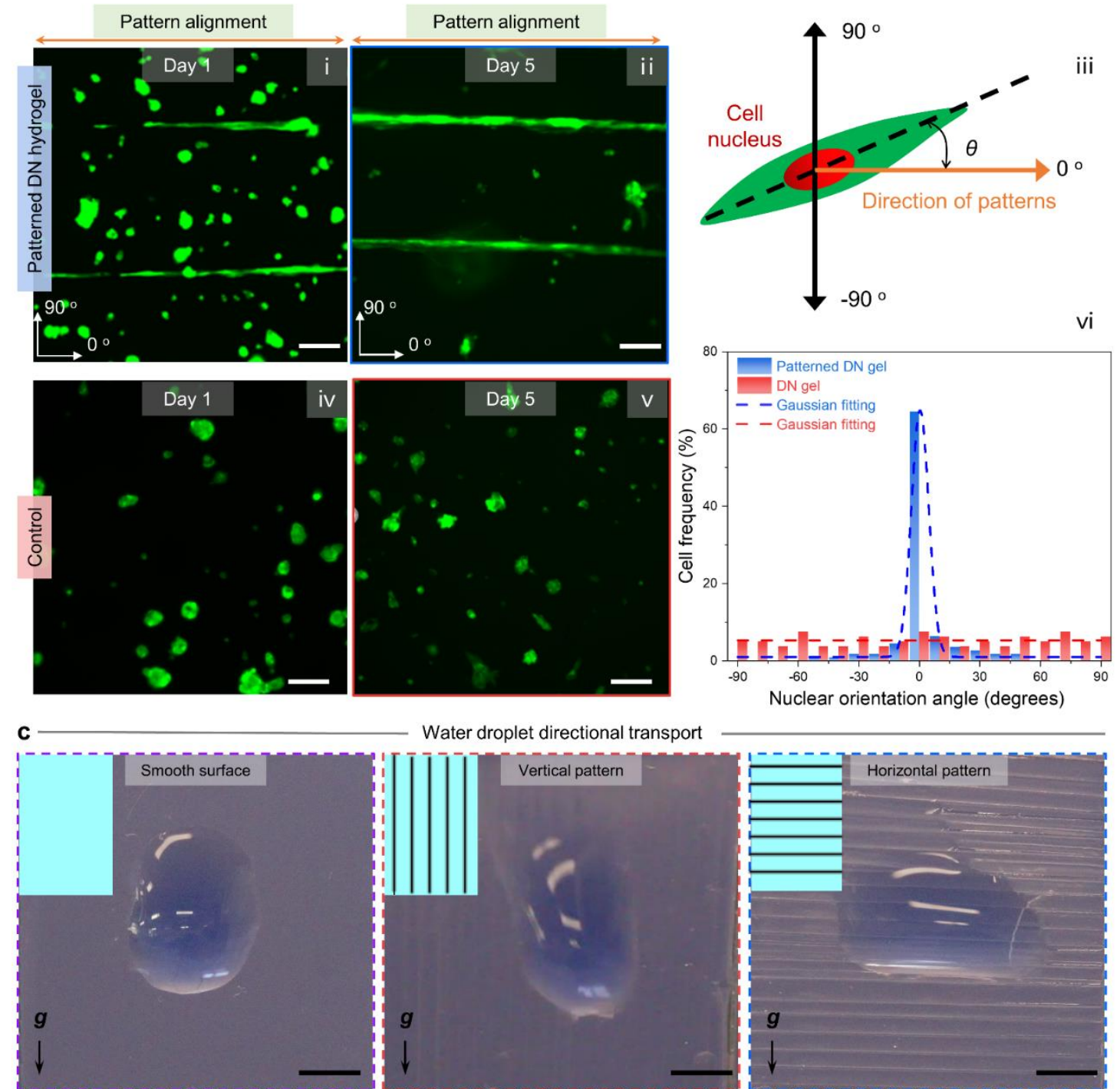

Water droplet directional transport
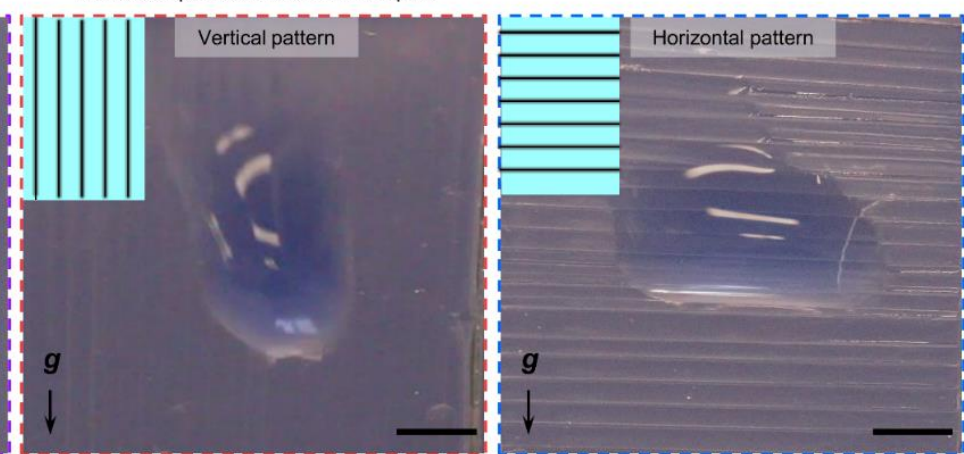

Fig.5| Specific applications of the micropatterned DN hydrogels. a, Optical images of DN hydrogels with parallel patterns of PNIPAm. Inset, an optical image with ANS under UV light. Scale bar, $1 \mathrm{~cm}$ (left), $500 \mu \mathrm{m}$ (right). b, Fluorescence images showing that the micropatterns guide cell orientation when cultured on the DN gel surface; myoblast cells (C2C12) labeled with a green fluorescence protein (GFP) were cultured on the PNIPAmpatterned DN hydrogel surface and smooth DN hydrogel surface (control) at $37^{\circ} \mathrm{C}$. Schematic diagram illustrating the procedure used to characterize the nuclear orientation angle between the pattern alignment direction. Quantification of the orientation of cells on 
518 patterned hydrogel and control hydrogel surfaces. Scale bars, $100 \mu \mathrm{m}$. c, Optical images

519 to show different morphologies of water droplets on DN hydrogel surfaces with different 520 anisotropic micropatterns. Dyed water droplet volume was $10 \mu \mathrm{L}$. The letter " $\mathrm{g}$ " and arrow 521 indicate the direction of gravity. NIPAm 1.0 M was used for a-c. Scale bars, $5 \mathrm{~mm}$. 


\section{Supplementary Files}

This is a list of supplementary files associated with this preprint. Click to download.

- Supplementarylnformation.pdf

- SupplementarymovieS1.avi

- SupplementarymovieS2.avi

- SupplementarymovieS3.avi

- SupplementarymovieS4.avi

- SupplementarymovieS5.avi 